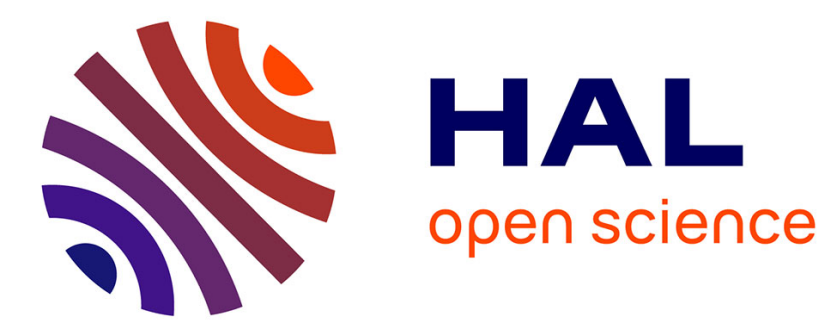

\title{
L'usage et ses modèles : quelques réflexions méthodologiques
}

Joëlle Le Marec

\section{To cite this version:}

Joëlle Le Marec. L'usage et ses modèles: quelques réflexions méthodologiques. Spirale - Revue de Recherches en Éducation , 2001, 28. sic_00000132

\section{HAL Id: sic 00000132 \\ https://archivesic.ccsd.cnrs.fr/sic_00000132}

Submitted on 21 Sep 2002

HAL is a multi-disciplinary open access archive for the deposit and dissemination of scientific research documents, whether they are published or not. The documents may come from teaching and research institutions in France or abroad, or from public or private research centers.
L'archive ouverte pluridisciplinaire HAL, est destinée au dépôt et à la diffusion de documents scientifiques de niveau recherche, publiés ou non, émanant des établissements d'enseignement et de recherche français ou étrangers, des laboratoires publics ou privés. 


\title{
L'usage et ses modèles : quelques réflexions méthodologiques
}

\author{
Joëlle Le Marec \\ Ecole Normale Supérieure Lettres et Sciences Humaines, Lyon \\ Section Art et Sciences de la Communication
}

Mots-clés : usages, représentations sociales, technique, musées, cédéroms, réseaux, communication

Résumé en français : Cet article tente propose de discuter de la notion d'usage sur la base suivante: la notion d'usage s'est développée en sciences humaines dans un contexte très particulier: celui de la «résistance» à une lecture de l'insertion sociale des nouvelles technologies en terme de phénomène socio-économique et de rapports de pouvoir. Les démarches très qualitatives centrées sur les pratiques, sur la construction des significations et des savoirs, se sont multipliées ces dix dernières années. La notion de représentations sociales est selon nous au cœur de ce mouvement, elle place les phénomènes liés à l'usage dans le champ des savoirs sociaux. Pourtant, les implications du recours à la notion de représentations sociales n'ont pas toujours été poussées dans leur logique : on le sent clairement dans le détail des études empiriques qui ont été menées sur les TIC en milieu muséal. Cette notion déplace selon nous la manière d'aborder les usages à partir de questions propres à des disciplines, elle ouvre sur la complexité des phénomènes de circulation des savoirs sociaux.

Résumé en anglais : We try here to discuss how research about the uses of technologies moved in the last years, from questions like : do we learn better with technologies? Or : how technologies determine evolutions of society? to other questions, about complexity of construction of social knowledge. Qualitative research, based on a diversity of local situations (for example what happens in musems, in libraries, at home?), was very important in this evolution.

\section{Article}

La notion d'usage est assez peu associée, traditionnellement, à des problématiques didactiques. Mais depuis les années 80, avec la montée d'un champ de l'éducation dite « informelle »- ainsi nommé par opposition au champ de l'éducation formelle, en milieu académique -les médias et les nouvelles technologies de l'information et de la communication (NTIC) ont constitué de nouveaux terrains et de nouveaux objets pour des recherches sur les apprentissages et les modes de circulation des savoirs. Il se trouve que médias et TIC sont également des terrains et objets privilégiés en sociologie et en sciences de l'information et de la communication, où la notion d'usage occupe une place très importante pour désigner les rapports, à la fois sociaux et techniques, qui s'établissent entre les individus ou groupes sociaux et les objets techniques. L'irruption continuelle de nouveaux objets techniques sur le marché a largement contribué au développement de la recherche sur les logiques de l'usage et le rapport à l'innovation. Or, la sociologie des techniques croise évidemment de son côté des questions liées à l'apprentissage et à la construction sociale des savoirs : les points de convergence entre les approches centrées sur la construction sociale des savoirs, et les approches centrées sur le rapport à la technique sont très nombreux.

L'entrée par les «nouvelles» technologies du moment (les cédéroms, les réseaux électroniques) ne constitue en réalité qu'un artéfact, un point de départ, dans le regroupement et la caractérisation d'un ensemble de phénomènes hétérogènes impliquant le recours à des 
dispositifs techniques. L'obligation de s'intéresser aux TIC crée un effet de nouveauté lié à la focalisation de la recherche sur des phénomènes qui tout simplement n'attiraient pas forcément l'attention auparavant. Le caractère de nouveauté est bien plus souvent dans l'attention que les chercheurs accordent à tel objet, que dans cet objet lui-même.

Nous allons dans cet article aborder la notion d'usage telle que nous l'avons saisie, tout au long d'un parcours de recherche de plus de dix ans dans laquelle elle est intervenue à de multiples reprises. Nous proposerons de réfléchir à cette notion à partir d'un concept qui a joué un rôle très important dans le champ de la didactique et dans le champ de la communication : les représentations sociales. Dans chacun de ces champs, les représentations sociales ont été comprises très différemment, ce que nous tenterons également d'analyser en conclusion.

Avec le recours à la notion d'usage, l'individu n'est plus avant tout saisi comme sujet épistémique, c'est-à-dire sujet apprenant face à des savoirs constitués comme représentations mentales : l'attention est focalisée sur les savoirs socio-techniques qu'il mobilise pour accéder à des savoirs. Ceux-ci continuent cependant à être posés comme des représentations mentales mises en forme sur des supports matériels (le plus souvent des documents). Par exemple, on se demandera comment l'individu se représente les phénomènes de digestion dans le but de lui proposer de nouvelles représentations mentales du phénomène. Mais si ces phénomènes sont abordés par le biais d'un didacticiel, on considèrera que la manipulation de ce dernier est un préalable obligé à l'appropriation de la proposition pédagogique : l'individu apprenant sera d'abord utilisateur de dispositifs pédagogiques, il devra utiliser de manière plus ou moins autonome des dispositifs et mobiliser pour ce faire une culture technique et médiatique : la maîtrise du dispositif apparaitra comme un préalable nécessaire à l'apprentissage ${ }^{1}$. C'est là une première entrée de la logique de l'usage dans le champ éducatif, par la sociologie des techniques et des sciences de l'information et de la communication.

De leur côté, les recherches sur les usages des TIC ont tiré parti d'un élargissement des enjeux bien au-delà des questions liées au rapport à l'innovation technique. Les questions liées à l'apprentissage, à l'activation des savoirs et à leur construction, à leurs modes de circulation et leurs ancrages locaux multiples dans des situations de communications sociales, sont continuellement réactivées dans toute observation ouverte des phénomènes liés à l'usage. De ce point de vue, les TIC sont à considérer moins comme des réalités empiriques, que comme une perturbation thématique des approches et des découpages disciplinaires. L'objet «NTIC » pose évidemment le problème du piège que constitue l'asservissement des problématiques de recherche à des enjeux politiques du moment, mais à cause de cela même, il oblige à des déplacements aussi bien en sciences de l'éducation, qu'en sciences de l'information et de la communication. Il intervient plus généralement dans la dynamique de toute approche en sciences qui tente de formuler des questionnements de recherche sur des articulations entre des niveaux, individuels et collectifs, entre des objets et des savoirs, entre ce qui se perpétue et ce qui se transforme. C'est pourquoi les mutations dont nous parlerons dans cet article sont de ce fait bien moins celles des phénomènes socio-techniques «objectifs » (si tant est qu'on puisse jamais parler en ces termes), que celles des approches développées dans le champ des représentations sociales et des pratiques. D'une certaine manière, les mutations sociales n'ont jamais à être postulées comme telles dans le champ scientifique dans la mesure où elles ne

\footnotetext{
${ }^{1}$ La mise en question croissante d'une conception des savoirs comme « contenus mentaux » qui existeraient indépendamment des situations sociales et des objets matériels dans lesquelles ils s'incarnent, est un mouvement quasi général. Mais les conséquences de cette mise en question n'ont cependant pas fini d'être tirées aux plans théorique et méthodologique.
} 
constituent pas des phénomènes observables, mais des interprétations a posteriori. Elles peuvent au mieux être saisies en tant que représentations inspirant les comportements et les interprétations des acteurs, qu'ils soient usagers ou chercheurs. A ce titre, elles génèrent certes des évolutions dans la recherche elle-même et ce sont ces évolutions qui engagent la nécessité d'être réfléchies ici et maintenant.

\section{Usages : notion plastique, affaire de cadrage}

Depuis quelques années, quantité de travaux ont approché le phénomène de l'insertion sociale des nouvelles technologies de l'information et de la communication, par des voies très variées. Les différences tiennent bien sûr, comme toujours, à des traditions disciplinaires. Par exemple, pour caricaturer les choses, la sociologie étudierait plutôt l'insertion progressive des usages dans des pratiques sociales à l'échelle de populations, l'anthropologie privilégierait l'exploration au cas par cas des comportements et des significations attachées à la conception et à l'usage de ces technologies, les sciences de l'information et de la communication tenteraient de mettre au point des approches intégrant la complexité des phénomènes dans leur dimension communicationnelle. Mais il nous semble que ces différences tiennent surtout au positionnement des études dans des lieux très variés, qui en «cadrent» très différemment la portée.

On étudie les nouvelles technologies dans les laboratoires universitaires, les ministères et les délégations ministérielles à la ville et à l'insertion, aux nouvelles technologies, aux arts plastiques, à la formation, dans les collectivités territoriales, dans les académies scolaires, dans les institutions culturelles (musées et bibliothèques en tout premier lieu), dans les départements marketing des sociétés de production multimédia. Ces études relèvent de la recherche, de l'évaluation, de la veille professionnelle, de l'engagement personnel ${ }^{2}$.

Les concepteurs multimédia de leur côté élaborent parfois des outils de travail intégrant des années d'une réflexion qui relèverait de la recherche si elle était développée au sein d'un institut de recherche ${ }^{3}$.

\footnotetext{
${ }^{2}$ Parmi les institutions culturelles, les musées et bibliothèques ont notamment mis en place des lieux et des réseaux où professionnels et universitaires peuvent développer des actions et des réflexions croisées, comme par exemple le Service Etudes et Recherches de la BPI, la cellule évaluation des expositions de la Cite des Sciences et de l'Industrie (CSI).

A la médiathèque de la Cité des Sciences et de l'Industrie, un département «Fonctions et publics spécifiques » coordonne une réflexion continue dans le but d'anticiper et de favoriser des évolutions dans les relations entre la médiathèque et ses publics (voir Agostini F.). 1994. «L'espace de la médiation : aventures en science », p. 301-320 in Science en Bibliothèque/sous la direction de F. Agostini. Paris, Le Cercle de la Librairie)

Des médiathécaires sont parfois continuellement en situation de susciter et d'expérimenter de nouveaux modes de relations aux publics dans des secteurs thématiques « sensibles » comme la santé.

Pour ce qui concerne les publications, la collection Etudes et Recherche de la BPI du centre Georges Pompidou, ou bien collection Bibliothèques des éditions du Cercle de la Librairie (par exemple : Rouhet (M.). 1996. Les nouvelles technologies dans les bibliothèques. Paris : éditions du Cercle de la Librairie) ou encore de nombreuses livraisons du Bulletin des Bibliothèques de France, sont caractéristiques de problématiques de recherche développées dans des cadres institutionnels des enjeux importants pour la dynamique et l'action professionnelles.

Pour un réinvestissement des études d'usages dans la réflexion professionnelle sur les pratiques informationnelles, voir : Le Coadic (Y.). 1997. Usages et usagers de l'information. Paris : Nathan Université.

${ }^{3}$ A la Cité des Sciences encore, le département Audiovisuel et Informatique des expositions expérimente continuellement la conception de produits interactifs innovants. Des bornes interactives comme «Le Livre d'Or », des sites sur réseaux électroniques en ligne comme « Conter ensemble » ou « masques : une collection virtuelle », cherchent ainsi à inventer des interactions qui sortent du modèle de la transmission institution---
} 
L'évaluation a également été très largement sollicitée, témoignant dans ce champ au moins de la légitimité des démarches mixtes qui «brouillent» largement logiques d'action et logiques de réflexion. A l'inverse, nombre de chercheurs sont totalement engagés dans des expérimentations techniques ou dans de la conception, la médiation, ou l'usage de ces technologies multimédia.

La problématique des usages tout à la fois souffre et bénéficie de ce dynamisme très particulier de la réflexion et de l'initiative qui touche tout ce qui concerne les nouvelles technologies de l'information et de la communication. Elle en souffre du fait du soupçon permanent de collusion entre la recherche et les instances de production, mais elle bénéficie de la confrontation permanente des questions de recherche et des objectifs institutionnels. En particulier, un des principaux problèmes posés par l'étude des usages, réside dans la grande difficulté de choisir parmi l'infinité des questions possibles, celles dont on devra faire l'hypothèse qu'elles s'avéreront en fin de compte les plus pertinentes et les plus stimulantes pour la réflexion et/ou pour l'action, sachant qu'il n'y a pas forcément possibilité d'intégrer les deux démarches, mais qu'inversement, elles ne sont pas nécessairement opposées (la réflexion serait du côté de la recherche tandis que l'action serait du côté du sens commun).

Plusieurs auteurs dans le champ des sciences de la communication et de l'information traitent de cette difficulté à anticiper les bonnes questions dans le cas de phénomènes qui sont vécus de trop près pour pouvoir en dégager les enjeux réels. Certains s'emploient à tenter d'organiser l'ensemble du champ en classant les démarches selon qu'elles sont centrées sur la réception ou bien sur la production. Ils s'efforcent de mettre ces approches en perspective les unes par rapport aux autres ${ }^{4}$, mais ils sont obligés, pour ce faire, de quitter le champ des phénomènes observables pour accéder à une analyse des analyses, nécessairement très distante des terrains où se produisent les phénomènes liés à l'avènement des nouvelles technologies de la communication et de l'information (NTIC).

Ces visions globales permettent d'adosser les phénomènes des usages, fondamentalement positif $f^{5}$ puisque «les gens» font toujours quelque chose avec, (avec la machine, l'objet, le dispositif dont on étudie l'usage) à des analyses qui en relativisent malgré tout la portée, soit en signalant le cadre limité que constitue la marge de manœuvre des usagers une fois que tout a été décidé en amont sans eux, soit en mettant l'accent sur l'importance des dispositifs de légitimation de certains usages au détriment d'autres, ou bien sur la pré-construction des usages dans des configurations de conceptions qui déterminent largement les conditions sociales de

>public. Il s'agit par exemple de favoriser (et de légitimer du même coup) des relations horizontales entre visiteurs, ou bien de créer des relations publics---->institutions pour l'élaboration de contenus culturels collectifs, ou bien encore de contribuer à la création d'une communauté de personnes liées par un intérêt thématique culturel, transcendant les catégories de « publics » et de « professionnels ».

\footnotetext{
${ }^{4}$ Voir notamment : Vitalis (A.) ed. 1994. Médias et nouvelles technologie: pour une sociopolitique des usages. Rennes : Editions Apogée.

Voir aussi le numéro 11-12 (1993) de la revue Hermès «A la recherche du public : réception, télévision, médias », Editions du CNRS

${ }^{5}$ En France en particulier, depuis Michel de Certeau, les usages ont été très vite étudiés en relation avec les pratiques culturelles au sens anthropologique, en tant que phénomènes directement intéressants par euxmêmes, même si leur portée s'est toujours limitée à la sphère des passions et des sociabilités privées, des tactiques et des ruses.

Voir : Perriault (J.). 1989. La logique de l'usage. Essai sur les machines à communiquer. Paris : Flammarion. Voir aussi: Toussaint (Y.), Mallein (Ph.).1992. «Diffusion, médiation, usages des technologies de la communication et de l'information », Culture technique, 24.
} 
l'accès aux dispositifs culturels, ou bien encore sur l'importance des discours d'accompagnement qu'on peut soupçonner d'orienter l'ensemble des dynamiques d'appropriation.

La question des usages est en effet souvent critiquée pour son caractère toujours trop «positif » (puisque les gens font tant de choses si intéressantes avec les dispositifs qu'on leur fournit ou qu'on leur impose, c'est donc que ceux-ci sont éternellement justifiés). La problématique de l'usage est peu à peu amenée à assumer des dimensions politiques et critiques, pour échapper au reproche de faire le jeu du marché et de la démagogie libérale. Le chercheur est bien souvent obligé de fournir tout à la fois les résultats de l'étude empirique des phénomènes observables auprès des usagers, et le commentaire critique ou politique qui les relativiserait aussitôt. La tradition de l'analyse critique des prises de position (écrites ou orales) d'acteurs «publics », et les analyses empiriques fondées sur le recueil du discours des acteurs «ordinaires» n'en continuent pas moins de s'affronter, dès lors qu'elles reproduisent au sein même des cultures disciplinaires l'impossible confrontation qu'elles tentent de dépasser. Les logiques politiques de la promotion ou de la prescription des usages sont analysées sur des plans fondamentalement hétérogènes aux logiques d'usages. Les premières font de préférence appel à des analyses de discours médiatiques ou de stratégies industrielles et économiques. Les secondes privilégient les études fondées sur le recueil des comportements et discours auprès des usagers individuels. La divergence est parfois purement méthodologique mais elle génère de toutes façons des désaccords insurmontables lorsqu'il s'agit de mettre en commun et d'utiliser les travaux ainsi réalisés. En effet, les premières prennent leurs distances par rapport aux discours produits par les acteurs, tandis que les secondes convoquent largement le discours des usagers sur le terrain. Les usages restent naturellement calés du côté de la réception du fait même de ces clivages théoriques et méthodologiques qui reproduisent finalement une structuration préexistante, économique et sociale. Ce qui est reproduit sans cesse dans ces préférences est une opposition bipolaire : logique offre/logique technique/logique économique d'une part, logique d'usage/logique sociale/sphères des intérêts privés d'autre part.

Les études d'usages ont pu ainsi contribuer à réifier la partition qu'elles mettaient pourtant explicitement en cause dans leur projet : les travaux sur la réception et les travaux sur la production sont effectués avec des approches et des enjeux scientifiques différents. La confrontation méthodologique est rarement possible de ce fait même : comment comparer par exemple des données empiriques sur les usages sociaux, et des analyses de discours d'accompagnement supposés représenter les stratégies de conception?

Les études d'usage accordent souvent en effet un rôle central à l'analyse des discours de personnes saisies dans des contextes où ils sont utilisateurs d'objets techniques. Les phénomènes liés à l'usage sont associés presque irrésistiblement à des discours d'usagers : en procédant préférentiellement de cette manière, on contribue à reléguer l'usager dans un pôle final, en bout de chaîne, puisqu'il ne produit plus rien que du discours. Même si on présuppose que ces discours vont de pair avec des comportements sociaux et des pratiques qui circulent hors de la sphère privée, les paroles dites à l'enquêteur ne pèsent objectivement d'aucun poids, elles ne sont porteuses d'aucune tension ou d'aucun risque qui les rendrait «actives » dans un autre contexte que celui de leur traitement par la recherche. Cette condition de l'usager comme étant statutairement, pour la recherche, un producteur de discours concernant un objet, est selon nous un artefact des études d'usage. Elle a été absolument nécessaire dans une vision un peu militante pour constituer les usagers en individus concrets, interlocuteurs sociaux potentiels puisqu'ils sont des interlocuteurs authentiques pour le chercheur. Par contre, on renforce ainsi 
très concrètement un rapport de force qui constitue l'usage en phénomène de réception pour des objets produits ailleurs.

Pourtant, la notion d'usage s'était largement développée en réaction à la conception consensuelle du processus d'innovation comme mécanisme linéaire, faisant intervenir une chaîne d'acteurs depuis les inventeurs et les promoteurs de l'innovation technique jusqu'aux utilisateurs, lesquels n'interviennent qu'en bout de course. Mais même si la réaction à cette conception a favorisé l'intérêt pour les usages en tant que phénomènes construits indépendamment des logiques de production, elle a contribué paradoxalement à renforcer la puissance du modèle linéaire devenu implicite. En effet, en s'attachant à valoriser l'importance des utilisateurs pour contrebalancer les représentations de la toute puissance des logiques de production, on s'inscrit à l'intérieur même du modèle qui les oppose. Fondamentalement, celuici persiste, d'autant plus qu'il se décline en une série de variantes (offre/demande, émission/réception) qui renforcent la réalité de la structuration qu'elles proposent. Or, ce modèle ne se superpose que trop bien à la vision du social comme étant in fine entièrement structuré et orienté par des rapports de pouvoir, et à la vision de la circulation des savoirs comme étant également structurée par des rapports de transmission. Le fait même de traiter la question des usages par la construction de la figure de l'usager, être incarné, sensible et pensant, socialement compétent et représenté par sa propre parole digne d'intérêt en tant que telle, est aussi un moyen de promouvoir la défense de la position du plus « faible » : la question des usages est prise encore une fois dans un schéma qui est en fin de compte soit un modèle de rapport de pouvoir, soit un modèle de transmission infiniment décliné.

Même si l'usage est désormais largement vu comme étant le fait d'allers et retours très complexes entre des logiques de production et des logiques de réception, il s'agit au fond toujours d'allers et de retours, c'est à dire d'un simple amendement du modèle linéaire et donc de son renforcement.

La redéfinition des phénomènes liés à l'usage comme étant des articulations entre logiques techniques et logiques sociales ${ }^{6}$ ouvre de nouvelles perspectives, mais trop souvent, le modèle linéaire travaille encore en sous-main cette reformulation.

Il apparait nécessaire de développer la réflexivité des approches pour échapper à ce problème, notamment par l'analyse, sur de longues durées, des travaux empiriques ayant mobilisé la notion d'usage.

Nous pensons qu'il faut remettre à plat la manière dont est en permanence réactivée la perception des usages sociaux comme phénomènes existant en réaction à quelque chose de prédéfini (des objets, des environnements objectifs, des logiques de production). La traditionnelle partition entre production et réception, stratégies et tactiques, bricolages et procédures professionnelles rationalisées, est bien sûr niée de façon consensuelle dans les multiples réflexions et recherches qui tentent de rendre compte de la complexité des logiques sociales et des articulations entre tous les phénomènes en jeu. Mais ce qui en résulte est souvent un simple aménagement de l'usage comme étant moins faible et plus riche que certains le supposaient, et de la production comme contenant elle aussi de l'usage. Ce qui doit être repensé est moins l'usage proprement dit que la pré-structuration de la recherche sur l'usage et la

\footnotetext{
${ }^{6}$ Voir J. Jouet (1997) « Pratiques de communication et figures de médiation : des médias de masse aux technologies de l'information et de la communication » in « Sociologie de la communication», sous la direction de P. Béaud, P. Flichy, D. Pasquier et L. Quéré, Réseaux, Centre National d'Etudes des Télécommunications, Paris.
} 
manière dont cette pré-structuration participe le plus souvent involontairement à un modèle conforté par ses aménagements.

Une réelle remise en cause du modèle linéaire dans l'analyse de l'usage passe par un décadrage radical des questions de recherche qui mobilisent la notion, et par conséquent, une approche méthodologique qui intègre absolument la contrainte d'éviter la prédétermination des phénomènes par ce modèle linéaires et par toutes ses déclinaisons, et l'engagement d'éviter la prédétermination des enjeux de recherche par la défense du pôle mineur, au plan scientifique et au plan social.

\section{Méthodologies d'approche des usages : de l'utilisation des objets techniques aux représentations sociales}

S'agissant d'un champ culturel, et même, comme dans le cas des bibliothèques, hyper-culturel ${ }^{7}$, il est impossible de référer l'étude des usages et de leurs significations aux seuls objets ou dispositifs techniques étudiés (lecture sémiologique de ces objets, et analyse de leur insertion dans les pratiques individuelles des usagers) comme cela a pu être le cas dans l'étude des technologies de la communication dans la vie quotidienne : le téléphone, le minitel, mais aussi le lave-linge, le four micro-ondes, etc.

Le champ culturel est en effet tout à la fois le champ de l'activation des relations entre sens et savoir, et le champ de l'activation des relations de l'individu à des collectifs de référence. L'importance de ces deux dimensions dans le champ culturel (les contenus et les rapports entre le collectif et l'individuel) permet d'éviter la tentation de hiérarchiser les phénomènes de l'usage en les étudiant d'abord en termes de rapports à la technique, pour ne s'intéresser qu'ensuite à l'appropriation de contenus. L'usage peut être envisagé comme la manière dont s'incarnent matériellement des relations au savoir.

Cette perspective est d'autant plus intéressante qu'au sein des institutions culturelles, du point de vue même des concepteurs dans des domaines où sont en jeu des phénomènes de circulation des savoirs et communications sociales, il est impossible de penser les usages en termes de simples rapports à la technique, ou phénomènes de réception. Qu'il s'agisse des dispositifs créés par des concepteurs pour des usagers, ou de situations de communications interpersonnelles directes entre des représentants de l'institution qui mettent en circulation des technologies et des membres du public qui les acquièrent ou en disposent dans des lieux dédiés, les phénomènes sont bien trop difficiles à modéliser selon les schémas de la transmission ou modèle offre/demande. Il serait mal venu que la recherche projette sur les logiques institutionnelles d'organisation ou de production, un simplisme qui n'existe plus guère que dans les discours d'accompagnement, lesquels ne représentent qu'eux-mêmes.

C'est par leur importance pour la compréhension de la manière dont s'actualisent des communications sociales structurées et les processus de construction des significations, que les usages peuvent être sortis du pôle «mineur» de la sociabilité privée et des représentations sociales prises comme conceptions naïves.

\footnotetext{
${ }^{7}$ Les sociologues de la lecture n'ont cessé de souligner le fait que la lecture incarnait la pratique culturelle savante par excellence, ce qui joue évidemment dans l'interprétation que les individus ont de toutes les pratiques liées au livre, à la lecture, aux bibliothèques.

Voir notamment les contributions de Martine Poulain, Jean-François Barbier-Bouvet et Michel Peroni à : Poulain (M.) ed. 1993. Lire en France aujourd'hui, Paris, éditions du Cercle de la Librairie.
} 
Dans une série d'études menées sur le multimédia culturel dans les musées, le croisement des regards individuels de professionnels sur les logiques de conception institutionnellement cadrées par des objectifs éducatifs ${ }^{8}$, et des regards individuels d'usagers sur les logiques d'usages, nous a permis de montrer que, à ce niveau tout au moins, les logiques d'usages intègrent un repérage et une reconnaissance des intentions de conception à partir desquelles ils se construisent, tandis que symétriquement, les logiques de conception se fondent partiellement au moins sur une anticipation constante des usages supposés ${ }^{9}$. Il suffit de saisir les unes et les autres par les mêmes méthodes pour déjà permettre des articulations qui dépassent la problématique du « décalage » entre conception et usage, ou de la confrontation de logiques entre organisations et individus. Nous réfléchissons actuellement, dans la foulée de ce type de recherche, à un de protocole permettant de recueillir auprès des concepteurs et des usagers non plus des données homogènes en termes de discours, mais des données homogènes en terme de productions concrètes et dispositifs créés ${ }^{10}$ : les concepteurs sont usagers et producteurs en tant qu'usagers, les usagers sont producteurs de dispositifs matériels privés (dispositifs de rangements, enregistrements, compilations, etc.) qu'ils peuvent même mettre en circulation, comme ce passionné d'art rencontré dans nos enquêtes, qui fabrique à l'usage d'une association à laquelle il appartient une encyclopédie, des cartes postales, des logos, des recueils d'images, en désossant ses cédéroms de musées.

$\mathrm{Au}$ fil des études réalisées à la cité des Sciences dans un premier temps sur les bornes interactives, puis à domicile sur les cédérom de musées, puis dans une variété de lieux publics sur les réseaux en milieu culturel, sont apparues des dimensions de l'usage qu'il semble désormais impératif de restituer dans la mesure où elles ancrent le phénomène non plus dans un univers purement discursif et individuel en réaction à tel objet particulier, mais dans la dynamique de circulation tout à la fois publique et privée des savoirs sociaux.

Dans un premier temps, dans la section qui va suivre, nous reviendrons sur les méthodologies en partant des approches qui font dériver l'usage de l'utilisation, pour aller vers une prise en compte méthodologique de l'usage comme représentation sociale.

Dans un second temps nous détaillerons des dimensions de l'usage qui sont apparues au fil de nos propres études et qui remettent en cause la stricte dépendance des discours à des objets empiriques particuliers : cédéroms, bornes interactives, sites. Cette opération se réalise non pas

\footnotetext{
${ }^{8}$ C'est le cas de la cité des Sciences et de l'Industrie

${ }^{9}$ On se réfère ici à deux ensemble de travaux, d'une part une série d'analyses des usages des bornes interactives à la cité des Sciences et de l'Industrie et au Musée d'Orsay, et d'autre part l'analyse actuellement en cours des discours de concepteurs de cédéroms culturels, en complément d'une étude des usages des cédéroms de musées. Voir : Le Marec (J.). 1993. «L'interactivité, rencontre entre visiteurs et concepteurs », Publics et Musées, 3.

Goldstein (B.), Le Marec (J.), Topalian (R.), Pouts-Lajus (S.). 1996. Interactifs : fonctions et usages dans les musées. Paris : Direction des Musées de France

Le Marec (J.). 1997. «Le multimédia dans les musées, valorisation du singulier et représentations du tout », in ICHIM 97, Actes de la 4ème conférence internationale sur les hypermédia et l'interactivité dans les musées. Archives § museum informatics Europe/Musée du Louvre, Paris 3-5 septembre 1997.

Davallon (J.), Gottesdiener (H.), Le Marec (J.). 1997. Approche de la construction des usages des cédéroms culturels liés aux musées. Paris : Direction des Musées Nationaux et Réunion des Musées Nationaux/SaintEtienne, CEREM, Université Saint-Etienne.

${ }^{10} \mathrm{Il} \mathrm{s}$ 'agit d'une recherche en cours, en réponse à un appel d'offre de la BPI et de la DLL sur les pratiques de lecture écriture liées aux réseaux, coordonnée par Davallon, Despres-Lonnet, Jeanneret, Le Marec et Souchier. Dans le cadre de laquelle Igor Babou est moi-même effectuons une étude spécifique sur les pratiques liées aux réseaux dans une bibliothèque, à partir des discours et des objets textuels recueillis auprès des personnels et des usagers.
} 
en faisant remonter en généralité les résultats de chaque étude portant sur tel objet particulier, mais tout au contraire, en faisant dépendre très étroitement et très concrètement les usages non plus seulement des objets, mais des contextes très précis dont ils font partie.

Par ailleurs, nous développons actuellement une perspective de recherche personnelle qui n'en est qu'à ses débuts et dans laquelle nous souhaiterions cette fois décadrer l'usage, non plus seulement par rapport aux objets techniques empiriques, mais par rapport à la production de discours comme manifestations typiques de la réception.

Les phénomènes liés à l'usage ont longtemps été référés à des objets techniques particuliers : PC, minitel, écran-clavier, cédérom, borne interactive, etc. Il semble toujours tomber sous le sens de commencer par les objets dans la détermination de ce qui fait usage, en faisant dériver celui-ci des phénomènes liés à l'utilisation. Cependant, surtout dans le cas des réseaux, il faut mettre en cause le support initial de la nouvelle technologie dont il est question. Même s'il est important de savoir comment les utilisateurs tapent sur le clavier, cette donnée d'observation est évidemment insuffisante pour savoir ce qu'ils fabriquent au juste. Si l'objet génère un niveau homogène d'activités observables par ses utilisations, lesquelles sont reliées aux usages, il est cependant fort possible qu'avec les réseaux, le rapport à l'objet technique comme préalable phénoménal obligé dans l'analyse des usages soit désormais remis en cause, et oblige à une radicalisation d'une dynamique qui était à l'œuvre depuis 10 ans dans la recherche. La réalité la plus sensible et la plus génératrice de phénomènes observables est bien moins l'univers de l'objet lui-même que l'univers des représentations d'usages qu'il génère. On se trouve à un moment que la recherche sur les usages s'est préparée à affronter: l'inversion entre les phénomènes objectifs et les représentations, dans la conception même de toute la recherche sur la question. Ce ne sont plus seulement les objets qui constituent la réalité de référence, mais l'univers des représentations en tant qu'occurrences concrètes, dans les discours et les pratiques, de savoirs et d'imaginaires qui sont en circulation permanente. Les représentations, largement imprévisibles et difficiles à cerner, constituent la réalité de référence des usages, lesquels se manifestent partiellement par des phénomènes objectifs observables. De ce point de vue, les objets techniques «en fonctionnement 》 (au sens le plus large) sont eux-mêmes des éléments constitutifs des systèmes de représentations.

A côté des études basées sur l'observation d'utilisateurs face à l'écran, la sociologie des usages a considérablement développé les approches par entretiens longs qui permettent de contextualiser un rapport à la machine dans des corps de pratiques et des systèmes de représentations. Rappelons ici pour mémoire que les représentations sociales ont été définies par Moscovici (1961) comme des savoirs sociaux, à la charnière de l'individuel et du collectif, qui sont produits et mobilisés au cours d'interactions et dans des processus de communication, et qui ont une visée pratique. L'entretien est une technique privilégiée pour saisir ces phénomènes. Cependant, le problème central reste la thématisation du matériel discursif recueilli. Si ce qui est dit par les usagers est rapporté prioritairement aux objets techniques (on rapportera par exemple ce qui est dit par les visiteurs dans des enquêtes sur le minitel, l'informatique, les cédéroms, à des représentations du minitel, de l'informatique, des cédéroms). l'usage qui est saisi à travers l'observation et les discours est implicitement une actualisation de la représentation sociale de l'usage de telle ou telle machine. Plus on assimile l'usage au phénomène des représentations sociales, moins il est pertinent de pré-thématiser les discours. On peut en quelque sorte lâcher le guidon des manifestations objectives comme la manipulation des objets, pour tester ce que cela fait de réellement prendre au sérieux 
l'hypothèse selon laquelle les usages sont des savoirs sociaux dont il faut découvrir le sens sans le pré-constituer par la référence à l'objet ${ }^{11}$.

C'est essentiellement dans le mode d'interprétation des discours recueillis que se joue le changement de référence. Les résultats ne mettent plus forcément en priorité ce qui concerne l'objet, pour ensuite s'intéresser à l'ancrage social des rapports à l'objet. On peut tenter de repérer quels sont les thèmes des discours des enquêtés sans le référer systématiquement à l'objet qui intéresse initialement le chercheur (un cédérom, un ensemble de sites, etc.). On aboutit parfois à la découverte de nouveaux « objets ». L'objet de l'usage peut alors être une petite partie de l'objet matériel prédéfini par le chercheur comme étant l'objet technique dont il est question, ou bien un ensemble matériel plus large, ou un mixte de composants matériels et spatiaux : le PC, des fragments de cédéroms, des dossiers imprimés etc.

C'est ainsi que le contexte de l'usage, et les techniques développées par les personnes ont finalement changé de statut au fil de mes propres études d'usages des nouvelles technologies en milieu culturel.

Le contexte n'est pas un environnement objectif éventuellement filtré par les représentations sociales, dans lequel prendraient place les phénomènes de l'usage tels qu'ils se manifestent par des comportements et des discours en situation. Il est un contenu de représentations. En analysant la manière dont les usages sont liés à des contextes, $\mathrm{j}$ 'ai été amenée à basculer le point de vue : dans la mesure où les usages peuvent être vus comme des représentations en actes qui s'actualisent dans des situations qu'elles contribuent à créer, orienter ou modifier, le contexte est une partie intégrante de l'usage. On le voit particulièrement dans la comparaison entre usages d'interactifs dans les espaces d'exposition, et usages de cédéroms de musées chez soi. La «différence» entre les deux ensembles de résultats n'est pas seulement une différence entre deux dispositifs techniques et une différence entre deux lieux, qui seraient répercutées sur ce que font les gens et ce qu'ils pensent qu'ils font. Ce que font les gens et ce qu'ils disent qu'ils font rendent apparentes des articulations très étroites entre les dispositifs, les lieux et les situations, un peu comme les anamorphoses multipolaires changent la vision d'une carte en y intégrant concrètement la valeur de ce qui y est vu. Par exemple, c'est par le biais des usages des interactifs que les espaces d'exposition à la cité des Sciences et de l'Industrie sont apparus pour la première fois comme étant des environnements bien réellement communicationnels, parce que les utilisateurs de bornes interprétaient leur propre activité, dans ce contexte, comme étant des activités de communication : ils interprètent leur propre activité comme étant un «contenu » au sens pédagogique et culturel, le plus pertinent des contenus dans la mesure où il s'articule directement à « ce que les concepteurs ont voulu me faire faire ».

Il en va tout autrement pour les cédéroms consultés chez soi, qui sont très rarement vus comme des moyens de communication avec des auteurs, mais comme des objets entièrement privés $^{12}$. Plusieurs facteurs entrent en jeu dans ce décalage, le changement de contexte bien sûr (au musée ou chez soi), la nature des objets (bornes informatiques et cédéroms) et la nature des contenus (scénarios souvent réalisés dans un contexte de diffusion des sciences dans un cas, navigation dans des fonds, émanant de musées de collections dans l'autre cas).

\footnotetext{
${ }^{11}$ J'ai développé ces aspects dans ma thèse. Voir Le Marec (J.). 1996. Les visiteurs en représentations : l'enjeu des évaluations préalables en muséologie. Université de SaintEtienne.

${ }^{12}$ Voir le Marec (1998).
} 
Toutes ces différences sont articulées dans des configurations complexes où les choses « vont ensemble » bien plus qu'elles ne sont la cause ou l'effet.

On aboutit à une inversion possible : le problème de la montée en généralité des résultats de recherche sur les usages étudiés pour une grande variété d'environnements, de lieux, de dispositifs, peut se résoudre bien autrement qu'en attendant indéfiniment de voir apparaître ce qui serait transversal et permanent au-delà des cas de figures multiples. On peut tout au contraire, au fil des recherches, faire l'hypothèse que les usages sont une notion qui permet de voir la construction des contextes sociaux, en tant que représentations sociales intégrant des éléments matériels et situationnels. La montée en généralité se fait sur la portée de la notion comme variété des représentations sociales intégrant des comportements et des savoirs, mais aussi, nécessairement, "pour de vrai » en quelque sorte, des objets matériels, des lieux concrets, etc.

De même, les techniques mises en œuvre par les utilisateurs de machines pour utiliser cellesci en tant qu'outils techniques sont traditionnellement perçues dans la recherche sur les usages comme étant liées immédiatement aux contraintes et potentialités d'utilisations des objets. Elles sont donc perçues comme relevant du mode d'emploi, c'est-à-dire le plus court chemin entre l'individu comme opérateur de comportements, et la machine. Et pourtant, là encore, un élargissement de la notion d'usage comme s'adossant à des représentations permet de considérer les façons de faire avec l'outil non pas comme étant avant tout des réactions à la machine, mais comme étant des élaborations parfois très complexes. Nous en verrons un exemple plus bas avec l'utilisation des grossissements pour les cédéroms de peinture.

\section{Les dimensions de l'usage : projets, contextes, techniques}

Nous faisons l'hypothèse que l'usage est une notion plastique, ouverte et complexe qui peut intégrer au moins trois autres dimensions fondamentales, les projets, les contextes, les techniques, ces trois dimensions s'appuyant largement sur les représentations sociales. .

\section{Le projet comme dimension de l'usage}

Les personnes que nous interrogeons dans le cadre des études d'usage ne sont presque jamais «naïves» quant à leur propre implication dans un projet culturel et social de diffusion des nouvelles technologies. La prise en compte de l'objet comme étant toujours un projet est centrale, elle structure une articulation possible entre la réaction des personnes aux discours d'accompagnement, et leur implication toute personnelle par rapport à leurs propres intérêts et à leur propre histoire. En tant que possesseur de cédérom par exemple, on est enrôlé d'une manière ou d'une autre dans un projet culturel et sociétal, et il n'est pas impossible que cette qualification de l'objet comme projet social général contribue à structurer l'usage personnel comme étant lui-même un projet d'usage. 
On peut ainsi d'ores et déjà s'appuyer sur certains des résultats d'une étude sur les cédéroms culturels montrant la puissance des logiques de projet et la manière dont elles déterminent le rapport à l'objet et à son contenu ${ }^{13}$.

Le projet d'usage s'articule à un projet de vie, comme dans le cas de retraités aisés qui sont dans des démarches d'auto-formation très ambitieuses, et de requalification de l'ensemble de leurs liens sociaux, familiaux, associatifs, institutionnels par le biais de nouvelles pratiques, mais aussi dans le cas de jeunes actifs poussés à associer de plus en plus leurs engagements professionnels et leurs investissements personnels, avec un degré d'exigence très fort dans la recherche d'une cohérence globale qu'ils attendent aussi des contenus proposés par les nouvelles technologies.

Dans une récente étude d'usages culturels d'internet on retrouve également des projets d'usages associés à des projets de vie, mais cette fois de la part de jeunes chômeurs ou de personnes qui se ressentent marginalisées ou en passe de l'être. Pour la plupart non équipés en informatique et ne disposant guère des moyens de l'être, ils utilisent les bornes et les réseaux dans des lieux publics de consultation, avec le souci d'articuler deux temps contradictoires de l'usage : le court terme («l'ici et maintenant» de la consultation sur le lieu public) et le projet à long terme de réintégration de collectifs de référence et de formation personnelle.

Dans les deux cas, les récits biographiques mettent en scène des représentations de l'évolution et du changement, individuel et général, mais aussi des représentations de procédures formelles d'apprentissage, de pratiques informationnelles, de modes d'accès aux savoirs.

Or, ces représentations activent elles-mêmes des conceptions de l'institution culturelle, des références culturelles, des métiers et des spécialités, des critiques des médias et de la médiation (les visites guidées, les émissions télévisées, les expositions, les nouvelles technologies, les propositions culturelles d'une manière générale), que l'on peut croiser avec les représentations que les «savants», les professionnels de tous ordres, se font des «ignorants » ou bien du public.

\section{L'intégration du contexte dans la construction des usages et dans la construction de l'offre}

Par la comparaison d'une série d'études indépendantes menées successivement à la BPI, dans des musées et expositions, à la maison, dans des magasins faisant office de lieux publics de consultation, dans des salles municipales, des écoles, des centres culturels, des salons temporaires, nous avons pris la mesure de ce que pouvait représenter dans la construction des usages, non seulement la nature particulière du dispositif informatisé étudié, ce qui était prévisible, mais aussi le contexte d'utilisation, public ou privé, institutionnel ou totalement informel.

Ce contexte détermine bien sûr des conditions spécifiques d'utilisation des dispositifs étudiés. On ne fait pas la même chose avec un catalogue informatisé à la bibliothèque, des bornes muséographiques dans les musées, des cédéroms de consommation chez soi, des réseaux

\footnotetext{
${ }^{13}$ Voir encore : Davallon (J.), Gottesdiener (H.), Le Marec (J.). 1997. Approche de la construction des usages des cédéroms culturels liés aux musées. Paris : Direction des Musées Nationaux et Réunion des Musées Nationaux/Saint-Etienne, CEREM, Université Saint-Etienne.
} 
électroniques dans une variété de lieux et de configurations d'accès, gratuites ou payantes, libres ou assistées et contrôlées, légitimantes ou non.

Il détermine également, quoique moins fortement un cadrage de l'usage dans des pratiques sociales et culturelles préexistantes : la fréquentation des bibliothèques, la visite d'exposition, la lecture et les loisirs cultivés, l'auto formation et l'autodidaxie, la sociabilité familiale ou urbaine autour des objets culturels et des technologies de communication, l'intérêt systématique pour la nouveauté et la fréquentation des lieux «branchés», la circulation par achat, don, prêt et échange de nouveaux objets symboliques.

Ces pratiques peuvent cependant être à leur tour recadrées, recomposées, complexifiées par l'usage du nouveau dispositif, mais surtout, par la volonté de nombreux usagers de faire du nouveau avec le nouveau. Le discours d'accompagnement des nouvelles technologies de la communication intervient évidemment à ce niveau, mais dans la mesure où on se trouve dans le champ culturel par excellence, celui du livre et de la bibliothèque, il intervient dans un domaine déjà naturellement très chargé en prescriptions symboliques, c'est-à-dire en usages symboliques de références, tout aussi importants et prégnants que les usages effectifs. Ces discours implicites sont au moins de trois ordres :

- celui des instances politico-culturelles, rattachées notamment aux ministères, et dédiées aux nouvelles technologies

- celui, plus ou moins implicites, des missions institutionnelles de la bibliothèque publique, pris en charge et discuté à tous les niveaux par les personnels

- celui des usagers eux-mêmes sur leurs propres aspirations et leurs propres valeurs, plus ou moins disponibles pour prendre en charge ou jouer le jeu d'une utopie collective. Ils l'assument alors soit directement, soit en la raccrochant à des aspirations individuelles. Celles-ci peuvent par exemple tenter de recréer des liens sociaux transcendant les classes et les groupes de références classiques, multiplier les «secondes chances » et accéder à tout moment à des opportunités de changer d'état, de condition, de destin personnel, etc. Le contexte intervient alors très fortement, dans la mise en place de l'articulation entre l'individuel et le collectif. On l'a vu avec la comparaison des bornes muséographiques et des cédéroms culturels, il peut parfois y avoir un changement radical dans la nature de ce qui est fait à l'écran selon que l'on se trouve dans un lieu public ou chez soi.

Cette importance du contexte est probablement également importante dans la construction de l'offre. On peut l'étudier pour les déterminations que les cultures professionnelles et institutionnelles font peser sur la conception multimédia d'une part, et sur l'aménagement de modes d'accès et de formes de médiation impliquant ces nouvelles technologies d'autre part. On peut également l'étudier pour la part que la nouveauté peut prendre dans les réticences, ou tout au contraire, dans des dynamiques volontaires de création portées par l'acceptation d'une certaine perte de maîtrise et imprévisibilité de ce qui peut advenir du fait de la mise en accès des nouvelles technologies. En effet, ces technologies sont souvent l'occasion de réformes déjà plus ou moins annoncées depuis longtemps, dans l'ensemble du dispositif d'accès et de médiation que constitue une bibliothèque. Elles sont l'occasion d'une reconstruction plus ou moins volontaire des contextes d'intervention professionnelle, où la part de création se paie d'une part d'imprévisibilité souhaitée ou redoutée.

\section{Techniques de l'usage}

L'usage a souvent été désigné comme un bricolage, construit à des fins tactiques en réaction ici et maintenant à l'impact d'un nouvel environnement ou d'un nouvel objet, la dimension stratégique étant réservée à la sphère de la production. 
Les activités manipulatoires peuvent être lues autrement que comme des façons de se débrouiller avec des objets techniques. Elles peuvent être elles-mêmes considérées comme des techniques inventées et mises au point par les acteurs pour «fabriquer» quelque chose avec les technologies, que ce soit pour soi-même ou pour d'autres que soi.

On pourrait prendre un exemple de ces techniques dans l'utilisation que font les utilisateurs observés dans une variété de situations et de contexte (borne interactive à Orsay, cédéroms de peinture consultée chez soi ou dans la librairie du Louvre) de la fonction «loupe» ou « détails» proposés par de nombreux scénarios. On peut en effet mettre en relation des variantes multiples de cette fonction comme technique du regard matérialisé dans son trajet à l'écran, depuis le simple parcours de l'image reproduite à l'écran au moyen de la souris utilisée comme pointeur, jusqu'à la plongée dans l'image active au moyen de cette même souris dans des cédéroms où les images sont entièrement numérisées. Si on peut parler de technique du regard, c'est dans la mesure où les personnes interrogées ont pratiqué et comparé plusieurs propositions dans les cédéroms, et acquis une «technique » de cette technique. Ils ont, dans le cas des visiteurs de la librairie du Louvre, perçu le caractère désormais «obligatoire » de cette fonction proposée à l'utilisateur et s'en servent pour évaluer les cédéroms d'art. Ainsi, sont bien distingués les cédéroms où l'on propose des agrandissements de certaines parties d'images de tableaux en cliquant sur une sélection préconstituée de zones actives, les cédéroms où l'image du tableau est entièrement active, les cédéroms qui proposent une fonction zoom.

La technique au sens de mode d'emploi cache finalement la technique au sens d'une combinatoire complexe entre la technique d'utilisation de l'objet technique, la technique de maitrise de nouvelles situations, la technique du regard. En ce sens, les techniques comme dimensions constitutives de l'usage sont bien des représentations sociales elles aussi.

\section{Réévaluation de quelques « problèmes » méthodologiques}

La pertinence scientifique et sociale des recherches empiriques portant sur les phénomènes liés à l'usage est continuellement discutée. Sont souvent désignés trois «problèmes »:

- le déplacement continuel de la notion dans le large spectre des représentations sociales comme phénomènes situés entre mythes et comportements

- la question de la difficile montée en généralité des études empiriques, qui constituent un fonds dont la taille croissante et la richesse génèrent des attentes toujours déçues de synthèses globales

- la variété des contextes dans lesquels est formulée la demande de connaissances sur l' usage (recherche, institutions culturelles, conception etc.)

A notre avis, les trois problèmes précédents constituent presque les indices, au plan méthodologique du fait que l'on n'ose jamais aller assez loin dans le lien très fort qui existe entre usages et représentations sociales. Ils désignent pourtant trois pistes intéressantes qui encouragent à penser encore plus les usages comme représentations, et qui permettent de plus de se servir des usages pour enrichir le concept de représentations.

En premier lieu, la plasticité de la notion d'usage est en soi intéressante : le déplacement entre «mythes» (les imaginaires) et comportements (les manipulations observables) n'est difficile à penser que s'il est modélisé comme un énième schéma linéaire qui irait 
de la surface des observables aux couches profondes des logiques inconscientes. L'usage est intéressant justement à condition de ne pas être à son tour décomposé en strates sur un mode analytique. Il n'est intéressant que s'il manifeste lui-même en permanence le fait que les phénomènes qu'il désigne sont des mixtes qu'on renonce au moins momentanément à décomposer pour les voir justement marcher en tant que mixte. On est là rigoureusement dans la même problématique que les représentations sociales. Par rapport à celles-ci, trop souvent considérés comme des contenus mentaux, les usages permettent en plus d'intégrer la demande institutionnelle de leur de prise en compte, car ils conservent le caractère très dynamique et opératoire de savoirs sociaux saisis en actes.

En second lieu, la montée en généralité n'est peut-être pas le destin le plus intéressant des études de cas. Elles devraient tout au contraire être examinées dans ce qu'elles ont de toujours différents. Les études de cas ne cessent de proposer des ouvertures théoriques pour une notion qui ne peut être que toujours en construction. L'usage pourrait de ce point de vue être un concept-instrument, un concept catalyseur pour continuellement mettre en question et rouvrir des catégories qui se figent toujours trop vite : le contexte par exemple, et les techniques.

\section{Conclusion}

Si l'on revient à notre point de départ, c'est à dire l'importance de la question des usages dans les problématiques éducatives, on peut faire apparaître un déplacement progressif des raisons qui ont motivé le recoupement usages/éducation : c'est en première approche une injonction sociale externe, l'intérêt obligé pour tout ce qui concerne les nouvelles technologies, qui a opéré une articulation entre les questions d'apprentissage et d'appropriation individuelle et sociale des savoirs d'une part, et les usages vus comme des rapports obligés à des dispositifs techniques de diffusion des savoirs d'autre part.

Mais de mon point de vue, et à partir de la réflexion méthodologique et théorique que j'ai pu développer sur les usages depuis de nombreuses années, cette première articulation purement thématique a fourni l'occasion de creuser un concept qui est commun depuis longtemps aux chercheurs en éducation et aux sociologues des usages, et qui est retravaillé en profondeur à la faveur de ce croisement d'approches : les représentations sociales.

Les représentations sociales ont à une époque constitué en didactique une ouverture importante dans l'étude des «conceptions des apprenants », dans la mesure où elles ont posé les situations éducatives comme des situations de communications sociales, et de prendre en compte les apprenants comme des individus socialement situés, ayant des motivations toutes autres que le simple désir d'apprendre. Les représentations sociales ont donc décentré les recherches en didactique, depuis une conception des savoirs vus comme des contenus mis en forme mentalement chez le sujet épistémique, vers une conception des savoirs en actes et en situations chez des sujets sociaux, dans des communications où ces savoirs sont mobilisés et construits. Ces ouvertures sont allées de pair avec une attention croissante aux dimensions sociale de la construction des savoirs avec par exemple les concepts de pratiques de référence (Martinand) ou de transposition didactique, (Chevallard), puis de transposition médiatique (Triquet) ${ }^{14}$.

\footnotetext{
${ }^{14}$ Martinand J.-L. 1994. » La didactique des sciences et de la technologie et la formation des enseignants », Aster, 19.
} 
En sociologie des usages, les représentations ont permis, presque à l'inverse, de prendre en compte la richesse des savoirs mobilisés dans l'étude du rapport entre des individus et des machines. L'objet de cet article a été précisément cet enrichissement de la notion d'usage par des dimensions multiples qui dépassent totalement les questions d'utilisation.

En fin de compte, dans les deux cas, les représentations sociales ont servi à rendre nécessaire par des cheminements différents, aux plans méthodologiques et théoriques, l'articulation précise et détaillée des savoirs, des objets, et des pratiques. Ce qui est en jeu, en fin de compte, est bien un recadrage théorique des phénomènes socialement prédéterminés comme relevant initialement soit de l'éducation, soit du rapport aux objets techniques.

Bibliographie :

Clément (P.).1994. «Représentations, conceptions, connaissances », p. 7-38 in Conceptions et connaissances, sous la direction de A. Giordan, Y. Girault et P. Clément. Berne : Peter Lang. Davallon (J.), Le Marec (J.). 2000. «L'usage en son contexte. Sur les usages des interactifs et des cédéroms des musées », Réseaux, 10

Jodelet (D.). 1984. Les représentations sociales : phénomènes, conceptions et théories » in Psychologie sociale, sous la direction de S. Moscovici, Paris : PUF.

Jouet (J.). 1997. «Pratiques de communication et figures de la médiatisation : des médias de masse aux technologies de l'information et de la communication » in Béaud (P.), Flichy (P.), Pasquier (D.), Quéré (L.). eds. Sociologie de la communication. Paris : CENT.

Le Coadic (Y.). 1997. Usages et usagers de l'information. Paris : Nathan Université Le Marec (J.). 1996. «Ecran du regard, regards à l'écran » in Gervereau (L). ed. Peuton apprendre à voir ? Paris : L'image/ENSB.

Le Marec (J.). 1998. «Interactivité et multimédia : lieux communs revisités par l'usage » in Rencontres Médias 2 , Paris : éditions du centre Georges Pompidou, collection la BPI en Actes ;

Moscovici (S.). 1961. La psychanalyse, son image et son public. Paris : PUF, réed. 1976 Perriault (J.). 1989. La logique de l'usage: essai sur les machines à communiquer. Paris: Flammarion

Toussaint (Y.), Mallein (Ph.). 1992. «Diffusion, médiation, usages des technologies de la communication et de l'information, Culture Technique, 24.

Vitalis (A.) ed. 1994. Médias et nouvelles technologies : pour une sociopolitique des usages. Rennes : éditions Apogée.

Chevallard Y. 1985. La transposition didactique : du savoir savant au savoir enseigné.

Grenoble : La Pensée Sauvage.

Triquer E. 1993. Analyse de la genèse d'une exposition de science, Thèse, Université Claude Bernard, Lyon 1. 